\title{
Process tomography of quantum memory in a Josephson-phase qubit coupled to a two-level state
}

\author{
MATTHEW NEELEY, M. ANSMANN, RADOSLAW C. BIALCZAK, M. HOFHEINZ, N. KATZ*, ERIK LUCERO, \\ A. O'CONNELL, H. WANG, A. N. CLELAND AND JOHN M. MARTINIS
}

\author{
Department of Physics and California NanoSystems Institute, University of California, Santa Barbara, California 93106, USA \\ *Present address: Department of Physics, Hebrew University, Jerusalem 91904, Israel \\ e-mail: martinis@physics.ucsb.edu
}

A quantum computer will require quantum bits (qubits) with good coherence that can be coupled together to form logic gates ${ }^{1,2}$. Superconducting circuits offer a novel solution ${ }^{3-9}$ because qubits can be connected in elaborate ways through simple wiring, much like that of conventional integrated circuits. However, this ease of coupling is offset by coherence times shorter than those observed in molecular and atomic systems. Hybrid architectures could help skirt this fundamental trade-off between coupling and coherence by using macroscopic qubits for coupling and atom-based qubits for coherent storage $\mathrm{s}^{10,11}$. Here, we demonstrate the first quantum memory operation ${ }^{12}$ on a Josephson-phase qubit by transferring an arbitrary quantum state to a two-level state ${ }^{13}$ (TLS), storing it there for some time, and later retrieving it. The qubit is used to probe the coherence of the TLS by measuring its energy relaxation and dephasing times. Quantum process tomography ${ }^{2,14}$ completely characterizes the memory operation, yielding an overall process fidelity of 79\%. Although the uncontrolled distribution of TLSs precludes their direct use in a scalable architecture, the ability to coherently couple a macroscopic device with an atomic-sized system motivates a search for designer molecules that could replace the TLS in future hybrid qubits.

In quantum computation, coupling atomic qubits over macroscopic distances is a long-standing technological challenge. In ion-trap architectures, qubits are physically moved to regions where they can be positioned close to each other and coupled electrostatically ${ }^{15}$. Approaches based on cavity QED eliminate the difficulty of moving atoms, but instead use a resonant electromagnetic cavity to couple over macroscopic distances via guided photons $s^{5,6,16}$. Several recent proposals meet this challenge using further novel approaches ${ }^{10,11,17,18}$.

Superconducting wires are a natural medium for coupling between macroscopic and atomic states because currents and voltages obey quantum mechanics over length scales from macroscopic to atomic dimensions. At the macroscopic scale, the coupling remains coherent because superconductors have small dissipation. At the atomic scale, coupling is possible because the tunnel junction has a dielectric thickness $\sim 2 \mathrm{~nm}$ that approaches atomic size. When an atom carrying a single elementary charge moves by one atomic bond length inside such a tunnel junction, it produces a substantial image charge in the junction electrodes,
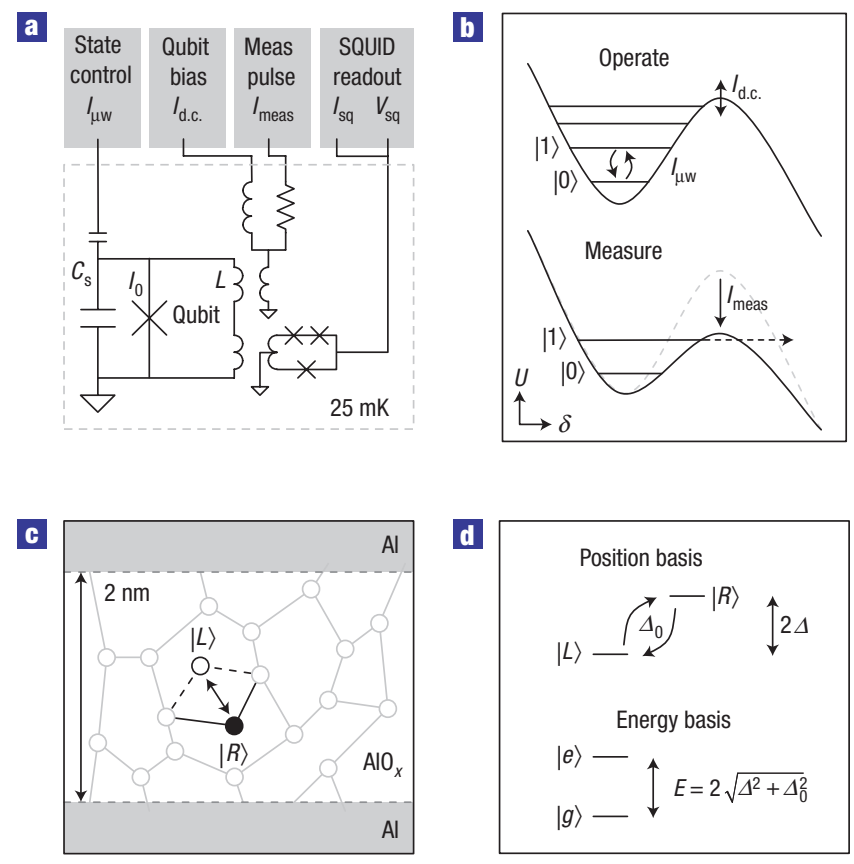

Figure 1 Register and memory qubits. a, Schematic diagram of the flux-biased phase qubit (register), a nonlinear resonator with $L=720 \mathrm{pH}$, shunt capacitance $C_{\mathrm{s}}=1 \mathrm{pF}$ and junction critical current $I_{0}=2 \mu \mathrm{A}$. SQUID: superconducting quantum interference device. $\mathbf{b}$, Plot of potential energy versus superconducting phase difference across the Josephson junction. The potential energy $U(\delta)$ has a cubic shape, and the qubit states $|0\rangle$ and $|1\rangle$ are the two lowest-lying quantum states in the potential well. During operation, microwaves $I_{\mu \mathrm{w}}(X$ and $Y$ rotations) and quasi-d.c. pulses $I_{\text {d.c. }}$ ( $Z$ rotations) manipulate the qubit state. During measurement, a rapid $(\sim 3 \mathrm{~ns}) I_{\text {meas }}$ pulse is applied to lower the potential barrier, allowing only the 1-state to tunnel out of the well. c, Representation of a TLS (memory qubit) in the amorphous $\mathrm{AlO}_{x}$ of the Josephson junction. Because the barrier material is disordered, some atoms can occupy two positions, labelled $|L\rangle$ and $|R\rangle$. d, The positional states are separated by an energy difference $2 \Delta$ and connected by a tunnelling energy $\Delta_{0}$. The energy eigenstates, the ground state $|g\rangle$ and excited state $|e\rangle$, are separated by an energy $E$. The dipole moment associated with charge motion between $|L\rangle$ and $|R\rangle$ couples to the currents and voltages in the qubit circuit. 


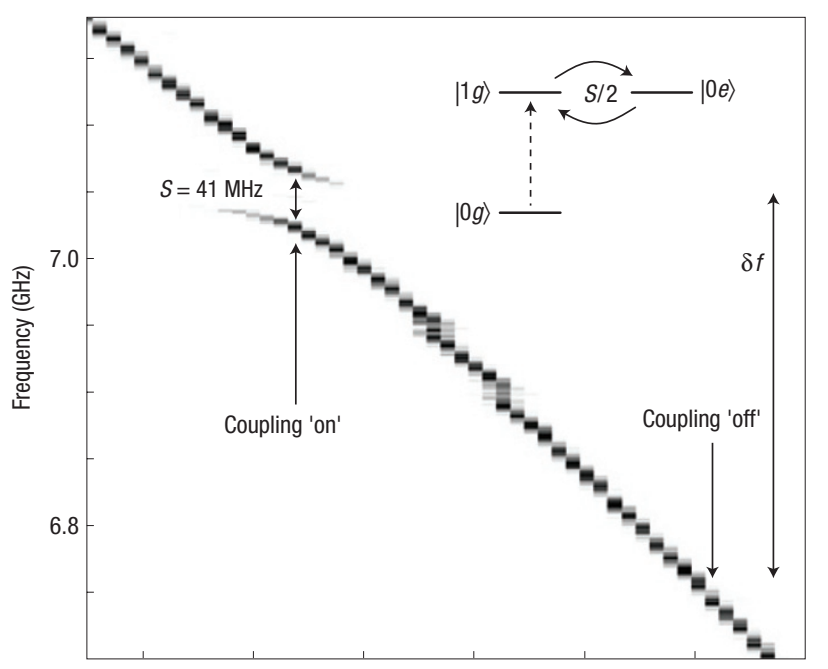

Flux bias (a.u.)

Figure 2 Modulation of the register-memory coupling. Spectroscopy plot showing register (phase) qubit excitation probability for microwave frequency versus flux bias. Probability $P_{1}$ of excitation to the $|1\rangle$ state is coded in greyscale. Changing the flux bias tunes the qubit frequency over a range $\sim 2 \mathrm{GHz}$. Coupling to the memory qubit (TLS) creates a splitting in the spectrum at $7.05 \mathrm{GHz}$. When on resonance, this coupling connects the register qubit excited state $|1 g\rangle$ and the memory qubit excited state $|0 e\rangle$, with a swap frequency equal to the splitting $S=41 \mathrm{MHz}$. When the register qubit is detuned from the memory qubit by $\delta f$, the coupling strength is reduced by $S^{2} / 4 \delta f^{2}$, effectively turning off the coupling.

coupling the atomic-scale motion to the macroscopic degrees of freedom of the currents and voltages in the circuit. We thus have a natural hybrid system: the atomic state in the junction is a 'memory' qubit capable of storing a quantum state, whereas the Josephson junction itself is a 'register' qubit capable of general logic operations and able to be coupled to other qubits over macroscopic distances.

Our register qubit, a flux-biased Josephson-phase qubit, is shown schematically in Fig. 1a. The qubit is a nonlinear inductor-capacitor resonator, whose resonant frequency can be tuned by varying the magnetic flux applied to the loop. The nonlinear resonator is well modelled at typical flux biases with a cubic potential, as shown in Fig. 1 b. The nonlinearity breaks the degeneracy (equal spacing) between adjacent energy levels, so that the application of microwaves produces transitions between only one pair of quantum states. Experiments are carried out so that only the two lowest-lying states are occupied; these constitute the qubit states $|0\rangle$ and $|1\rangle$. The qubit is controlled by applying pulses of magnetic flux. A quasi-d.c. pulse adiabatically changes the energy difference between $|0\rangle$ and $|1\rangle$, and the resulting accumulation of phase is equivalent to a $Z$-axis rotation of the Bloch vector ${ }^{2}$. A microwave pulse at the transition frequency coherently changes the occupation of the state, and corresponds to an $X$ - or $Y$-axis rotation ${ }^{19}$.

Our memory qubit is a two-level state (TLS) located inside the Josephson tunnel barrier, shown in Fig. 1c. A TLS is understood to be an atom, or a small group of atoms, that tunnels between two lattice configurations ${ }^{20}$. Such states are ubiquitous in amorphous materials, the disordered structure of which does not fully constrain the constituent atoms. The electric dipole moment inferred from measurements of these states is consistent with an atom carrying net charge $e$ moving by about one atomic bond length ${ }^{21}$. Because the barrier thickness is $\sim 20$ atoms, the induced charge on the electrodes from this motion is large, $\sim e / 20$.
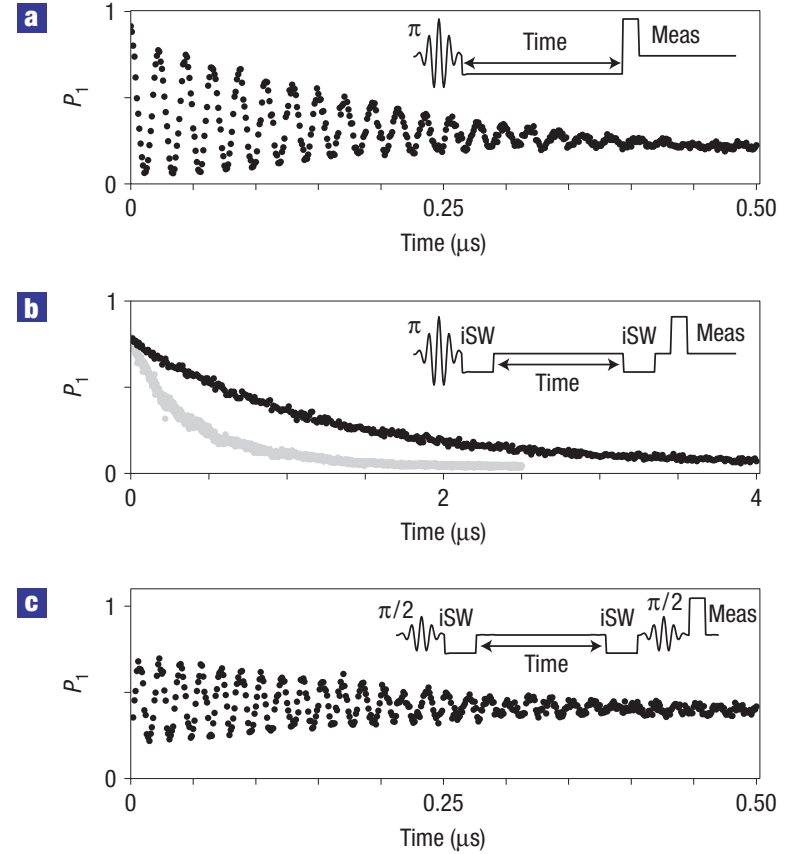

Figure 3 Time-domain interaction of the register and memory qubits. a-c, Register qubit excitation probability $P_{1}$ versus time for three pulse sequences, as represented in the insets. a, Free oscillations due to resonant coupling. The register qubit is excited with a $\pi$ pulse while detuned from the memory qubit, and then brought into resonance for a variable time before being measured. The excitation probability oscillates as the state swaps between $|1 g\rangle$ and $|0 e\rangle$. The position of the first minimum gives an iSWAP time of $12 \mathrm{~ns}$, in agreement with the spectroscopically measured splitting. The maximum probability decays to zero owing to $T_{1}$ processes in both qubits. $\mathbf{b}$, Energy decay of the memory qubit. The register qubit is excited while off-resonance, and then coupled to the memory qubit for 12 ns to transfer the state with an iSWAP. A second iSWAP later restores the memory state into the register. The resulting decay (black) gives the lifetime of the memory element $T_{1, \mathrm{mem}}=1.2 \mu \mathrm{s}$, which is longer than the register qubit lifetime $T_{1, \text { reg }}=0.4 \mu \mathrm{s}$ (grey). c, Ramsey fringes of the memory qubit. A superposition state $(|0\rangle+i|1\rangle) / \sqrt{2}$ is prepared in the register with $a \pi / 2$ pulse and transferred to memory. After variable time, the state is swapped back into the register and another $\pi / 2$ pulse is applied. The phase of the second pulse is swept with time, leading to Ramsey interference fringes that decay owing to dephasing of the state while in memory. The decay envelope gives $T_{2 \text {,mem }}=210 \mathrm{~ns}$.

TLS defects have been considered deleterious, as they provide extra quantum states to which the qubit may couple in an uncontrolled manner. A dense bath of these states is equivalent to dielectric loss, which decreases the lifetime of the qubit ${ }^{21}$. Decoherence from TLS defects can be removed by decreasing their number; this has been accomplished by reducing the junction area while shunting it with an external low-loss capacitor ${ }^{22}$.

Here, we demonstrate that a TLS can play a useful role as well. A previous experiment coupled a qubit to a TLS ${ }^{13}$, and theoretical work has suggested their use as memory elements ${ }^{12}$. In the present device, individual TLS states are well separated from each other in frequency, and the qubit lifetime is sufficiently long, that we can carry out precise gate operations between the register qubit and one TLS. The ground and excited energy eigenstates of this TLS, labelled $|g\rangle$ and $|e\rangle$, constitute the memory qubit. By adjusting the flux bias, the register and memory qubits are tuned into and out of resonance, effectively turning on and off their coupling. We have found that a TLS memory can have a reasonably long 

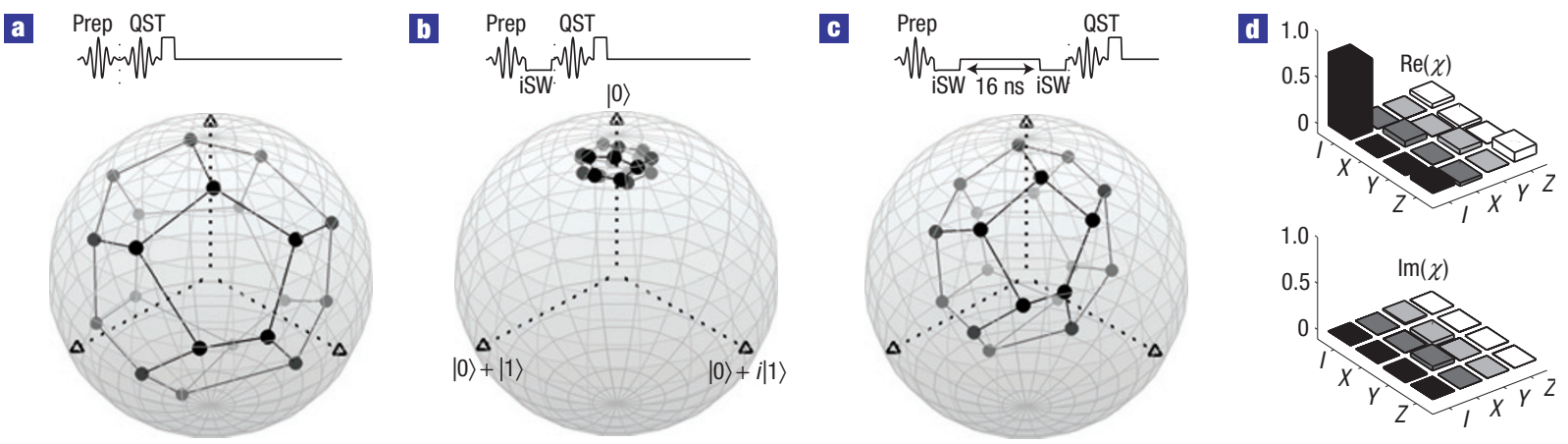

Figure 4 Process tomography of quantum memory. a-c, Register qubit state at three stages of the memory operation, using QST to reconstruct the states on the Bloch sphere. Circles indicate the states, whereas edges have been added to highlight the structure. Insets show the pulse sequences used at each stage. a, Initial states lying at the vertices of a dodecahedron on the Bloch sphere. $\mathbf{b}$, After the first iSWAP, the state has been transferred into the memory, and the register is left nearly in its ground state. c, After the second iSWAP, the state has been restored from memory into the register qubit. The structure of initial states is clearly preserved, although the amplitude has decreased owing to $T_{1}$ and $T_{2}$ decay processes in the memory during the $16 \mathrm{~ns}$ hold time. $\mathbf{d}$, Real and imaginary parts of the $\chi$ matrix for the memory operation, determined by comparing the initial states from a with the final states from $\mathbf{c}$. The large $l-l$ element indicates that the memory operation is a near-identity, as expected. From $\chi$, we compute a process fidelity of $79 \%$.

Table 1 Operator-sum representation of memory operation.

\begin{tabular}{lccrr}
\hline Weight (\%) & \multicolumn{1}{c}{$l$} & $\sigma_{x}$ & $\sigma_{y}$ & $\sigma_{z}$ \\
\hline 79.15 & 0.9976 & $-0.0052+0.0007 i$ & $-0.0010-0.0213 i$ & $0.0653+0.0034 i$ \\
12.92 & $-0.0576-0.0002 i$ & $-0.5925-0.0451 i$ & $0.0797-0.3164 i$ & 0.7329 \\
7.73 & $0.0089+0.0236 i$ & $0.0109-0.4065 i$ & 0.6685 & $-0.0882-0.6160 i$ \\
0.20 & $-0.0281-0.0010 i$ & 0.6940 & $0.0370-0.6670 i$ & $0.2668+0.0138 i$ \\
\hline
\end{tabular}

coherence time, and thus represents a good model system for future hybrid qubits.

The register qubit transition frequency $|0\rangle \rightarrow|1\rangle$ is first measured as a function of flux bias using spectroscopy, as shown in Fig. 2. The splitting at $\sim 7.05 \mathrm{GHz}$ is due to a TLS with coupling strength $S=41 \mathrm{MHz}$ that will be used as the memory qubit. We characterize the register qubit at the off-resonance frequency of 6.75 GHz, and find coherence times from standard energy decay, Ramsey and spin-echo sequences to be $T_{1}=400 \mathrm{~ns}, T_{2}=120 \mathrm{~ns}$ and $T_{2}^{*}=350 \mathrm{~ns}$, respectively. The measurement visibility is high, approximately $90 \%$. This phase qubit has a coherence time $T_{1}$ that is four times longer than previously reported, owing to the use of a new low-loss dielectric a-Si:H (refs 21,23) in the shunt capacitor.

To characterize the memory qubit, we first tune the register qubit off-resonance to $6.75 \mathrm{GHz}$ and excite it into the $|1\rangle$ state with a 16 -ns-long $X_{\pi}$ pulse. Then, a $Z$ pulse with 2 ns rise time moves the register qubit adiabatically into resonance with the memory qubit, effectively turning on the coupling. After waiting for time $t$, the register qubit state is measured. The resulting oscillations between the register and memory qubits, shown in Fig. 3a, have a $25 \mathrm{~ns}$ period that agrees with the coupling strength measured spectroscopically. In the rotating frame, the coupling is of the form $^{12}(S / 2)\left(\sigma_{x} \sigma_{x}+\sigma_{y} \sigma_{y}\right)$, so that the first minimum at $12 \mathrm{~ns}$ corresponds to an iSWAP gate ${ }^{24}$, which takes $|1 g\rangle \rightarrow i|0 e\rangle$ and $|0 e\rangle \rightarrow i|1 g\rangle$. The envelope of the oscillations between the register and memory decays more slowly than for the register qubit alone, indicating that the memory qubit has a longer lifetime.

The coherence of the memory qubit is directly measured using two iSWAP operations, as shown in Fig. 3b,c. We start by exciting the register qubit off-resonance, and then move it into resonance with the memory qubit for time $t_{\text {swap }}=12 \mathrm{~ns}$ to achieve state transfer into memory. After the register qubit is moved out of resonance to its starting frequency for a variable wait time $t$, it is then subjected to another iSWAP operation before measurement. Figure $3 \mathrm{~b}$ shows a plot of the measurement probability versus wait time; the exponential decay gives a qubit memory time $T_{1}=1.2 \mu \mathrm{s}$. Storing the quantum state in memory instead of the register increases its lifetime by a factor of three in our system, although this does not improve on the best $T_{1}$ times reported in other superconducting qubits. TLS lifetimes estimated from phonon radiation $^{25}$ depend on the electron-phonon coupling constant, which varies greatly from defect to defect. The measured lifetime is consistent with typical predictions that fall in the range of $10 \mathrm{~ns}-10 \mu \mathrm{s}$.

A similar Ramsey fringe experiment is used to measure dephasing, as shown in Fig. 3c. We first prepare the register qubit in the superposition $(|0\rangle+i|1\rangle) / \sqrt{2}$ with an $X_{\pi / 2}$ pulse, carry out the same iSWAP/hold/iSWAP sequence as before, and then execute a final $\pi / 2$ pulse with swept phase. The envelope of the Ramsey oscillations indicates a memory dephasing time $T_{2}=210 \mathrm{~ns}$.

This iSWAP/hold/iSWAP sequence is in fact a quantum memory operation for an arbitrary initial state in the register qubit. The first iSWAP transfers the state to the memory qubit, where it is protected from decoherence during the hold time; the second iSWAP then restores the register qubit to its initial state (up to a correctable $Z$ rotation). We characterize this memory operation using quantum process tomography ${ }^{2,14}$ (QPT), which involves preparing a spanning set of input states, carrying out the quantum operation and measuring the output with quantum state tomography ${ }^{22}$ (QST). The measured input and output states enable us to fully reconstruct the quantum memory process. The control sequence for QPT is similar to that for Ramsey fringes, with the microwave pulses generalized to create the initial states and to carry out QST on the final states. 
QST is carried out at three separate stages in the sequence, as shown in Fig. 4a-c. In Fig. 4b, after transfer to the memory qubit, the register qubit contains little amplitude of the initial state, as expected. The coherence of the memory operation is determined by comparing Fig. 4a-c, which shows only a small reduction in length of the Bloch vectors. Here, we have compensated for the $Z$ rotation arising from the two iSWAPs and the $295 \mathrm{MHz}$ detuning between the coupling 'on' and 'off' frequencies.

QPT gives us the $\chi$-matrix of the memory operation ${ }^{2}$, shown in Fig. 4 d. In this representation, the quantum operation acts on the input density matrix as $E(\rho)=\sum_{m, n} \chi_{m n} \hat{A}_{m} \rho \hat{A}_{n}^{\dagger}$, where $\left\{\hat{A}_{m}\right\}$ is some fixed set of basis operators, in our case the identity $(I)$ and Pauli $\sigma$-matrices. Diagonalizing the $\chi$-matrix leads to the operatorsum representation, which we write as $E(\rho)=\sum_{k} w_{k} \hat{E}_{k} \rho \hat{E}_{k}^{\dagger}$, where the operation elements $\left\{\hat{E}_{k}\right\}$ are linear combinations of the basis operators, and the weights $\left\{w_{k}\right\}$ give the probabilities of applying each operation element. Table 1 shows the operator-sum representation of the memory operation, giving the weight $w_{k}$ and the coefficients of the basis operators for each operation element $\hat{E}_{k}$. The dominant operation element is a near-identity, as we expect for a memory operation. The next-most dominant elements are primarily $\sigma_{z}$-like $\left(T_{2}\right.$ dephasing $)$ and $\sigma_{x^{-}}$and $\sigma_{y}$-like $\left(T_{1}\right.$ relaxation). The relative weights of these dephasing and relaxation elements are roughly as expected from the measured $T_{1}$ and $T_{2}$ times of the memory qubit, accounting for the overall length of the experiment $\sim 40 \mathrm{~ns}$. The simplest measure of fidelity, the trace overlap, gives a process fidelity of $79 \%$.

Errors in the memory operation can be divided into several categories, including tomography errors during state preparation and measurement, storage errors during the memory hold time and transfer errors during the iSWAPs. Tomography errors will be reduced by ongoing work to improve single-qubit performance through, for example, better materials and microwave pulse shaping. Storage errors represent the intrinsic $T_{1}$ and $T_{2}$ of the TLS memory qubit, and it may be possible to improve them by substituting a suitably engineered molecule. Finally, transfer errors that come from the register-memory interaction may be more difficult to reduce, although careful control of the qubit frequency in turning on and off the coupling should improve the transfer fidelity. Note that the transfer errors are independent of the memory hold time. Thus, after some crossover time $t_{c}$, the longer $T_{1}$ of the memory qubit offsets the transfer error, resulting in better overall memory fidelity than the register qubit alone. In this case, our analysis of the process tomography indicates that the TLS memory is useful beyond the crossover time $t_{\mathrm{c}} \sim 100 \mathrm{~ns}$.

Although a TLS was suitable for this initial proof-of-principle demonstration of quantum memory, their use in a quantum computer is unlikely because of their intrinsically random nature and limited coherence time. However, this experiment explicitly demonstrates a bridge between macroscopic and atomic-sized qubit states in a Josephson qubit, which motivates a search for a properly engineered atomic-scale memory qubit. Such a molecule should have a transition frequency in the $3-15 \mathrm{GHz}$ microwave range, a large electric dipole moment to couple to the capacitor, but a small motional dipole moment to minimize coupling to and loss from phonon radiation. With the increasing ability in the field of molecular electronics to fabricate designer molecules, a hybrid Josephson qubit with long coherence may be within reach.

\section{METHODS}

\section{FABRICATION}

The phase qubit used in this experiment was fabricated using procedures similar to those described in ref. 22 . We replaced the silicon nitride dielectric of that design with a new low-loss dielectric made from hydrogenated amorphous silicon to achieve $\sim 4$ times longer coherence time.

\section{QUBIT CONTROL}

Control signals for carrying out qubit manipulations are generated using a custom 2-channel 14-bit digital-to-analog converter with $1 \mathrm{~ns}$ waveform resolution. The output of the digital-to-analog converter is filtered with dissipative gaussian filters. Quasi-d.c. pulses for $Z$ rotations and measurement are sent directly to the qubit, whereas the microwave control signals are sent to a quadrature mixer to modulate the two quadratures of a microwave signal from a continuous-wave source. The input microwave frequency is set $100 \mathrm{MHz}$ above the qubit frequency, and a sideband modulation is used to mix this signal into resonance with the qubit. This prevents leakage at the carrier frequency from causing qubit transitions, thereby increasing the on/off ratio of the microwave control.

\section{STATE TOMOGRAPHY}

Three suitably chosen measurements are sufficient to completely characterize a single-qubit state, for example, projections along the $X, Y$ and $Z$ axes of the Bloch sphere. The phase qubit can only be measured along the $Z$ axis (distinguishing $|0\rangle$ and $|1\rangle)$, but rotations can be applied before measurement to effectively measure along other axes. For the present device, the visibility of the $|0\rangle$ and $|1\rangle$ states is different, that is, the probability of correctly identifying $|0\rangle$ is not equal to the probability of correctly identifying $|1\rangle$. For this reason, we carried out six measurements, one in each direction along the $X, Y$ and $Z$ axes. These six measurements can be combined to yield the Bloch vector of the state.

\section{Received 15 November 2007; accepted 26 March 2008; published 27 April 2008.}

\section{References}

1. DiVincenzo, D. P. The physical implementation of quantum computers. Fortschr. Phys. $\mathbf{4 8}$, 771-783 (2000).

2. Nielsen, M. A. \& Chuang, I. L. Quantum Computation and Quantum Information (Cambridge Univ. Press, Cambridge, 2000)

3. Devoret, M. H. \& Martinis, J. M. Implementing qubits with superconducting integrated circuits. Quantum Inform. Process. 3, 163-203 (2004).

4. You, J. Q. \& Nori, F. Superconducting circuits and quantum information. Phys. Today 58, $42-47$ (2005).

5. Sillanpaa, M. et al. Coherent quantum state storage and transfer between two phase qubits via a resonant cavity. Nature 449, 438-442 (2007)

6. Majer, J. et al. Coupling superconducting qubits via a cavity bus. Nature 449, 443-447 (2007).

7. Plantenberg, J. H., de Groot, P. C., Harmans, C. J. P. M. \& Mooij, J. E. Demonstration of controlled-NOT quantum gates on a pair of superconducting quantum bits. Nature $\mathbf{4 4 7}$, 836-839 (2007).

8. Niskanen, A. O. et al. Quantum coherent tunable coupling of superconducting qubits. Science 316, 723-726 (2007)

9. Steffen, M. et al. Measurement of the entanglement of two superconducting qubits via state tomography. Science 313, 1423-1425 (2006).

10. Tian, L., Rabl, P., Blatt, R. \& Zoller, P. Interfacing quantum-optical and solid-state qubits. Phys. Rev Lett. 92, 247902 (2004)

11. Sørenson, A. S. et al. Capacitive coupling of atomic systems to mesoscopic conductors. Phys. Rev Lett. 92, 063601 (2004).

12. Zagoskin, A. M., Ashhab, S., Johansson, J. R. \& Nori, F. Quantum two-level systems in Josephson junctions as naturally formed qubits. Phys. Rev. Lett. 97, 077001 (2006).

13. Cooper, K. B. et al. Observation of quantum oscillations between a Josephson phase qubit and a microscopic resonator using fast readout. Phys. Rev. Lett. 93, 180401 (2004).

14. Mohseni, M. \& Lidar, D. A. Direct characterization of quantum dynamics: General theory. Phys. Rev. A 75, 062331 (2007).

15. Kielpinski, D., Monroe, C. \& Wineland, D. J. Architecture for a large-scale ion-trap quantum computer. Nature 417, 709-711 (2002).

16. Boozer, A. D. et al. Reversible state transfer between light and a single trapped atom. Phys. Rev. Lett. 98, 193601 (2007)

17. Treutlein, P. et al. Bose-Einstein condensate coupled to a nanomechanical resonator on an atom chip. Phys. Rev. Lett. 99, 140403 (2007).

18. Nirrengarten, T. et al. Realization of a superconducting atom chip. Phys. Rev. Lett. 97, 200405 (2006)

19. Steffen, M., Martins, J. M. \& Chuang, I. L. Accurate control of Josephson phase qubits. Phys. Rev. B 68, $224518(2003)$

20. Phillips, W. A. Tunneling states in amorphous solids. J. Low Temp. Phys. 7, 351-360 (1972).

21. Martinis, J. M. et al. Decoherence in Josephson qubits from dielectric loss. Phys. Rev. Lett. 95, 210503 (2005).

22. Steffen, M. et al. State tomography of a capacitively shunted phase qubit with high fidelity. Phys. Rev. Lett. 97, 050502 (2006)

23. Liu, X. et al. Amorphous solid without low energy excitations. Phys. Rev. Lett. 78, 4418-4421 (1997).

24. Schuch, N. \& Siewert, J. Natural two-qubit gate for quantum computation using the XY interaction. Phys. Rev. A 67, 032301 (2003)

25. Carruzzo, H. M., Grannan, E. R. \& Yu, C. C. Nonequilibrium dielectric behavior in glasses at low temperatures: Evidence for interacting defects. Phys. Rev. B 50, 6685-6695 (1994).

\section{Acknowledgements}

Devices were made at the UCSB and Cornell Nanofabrication Facilities, a part of the NSF funded NNIN network. This work was supported by ARDA under grant W911NF-04-1-0204 and NSF under grant CCF-0507227.

\section{Author information}

Reprints and permission information is available online at http://npg.nature.com/reprintsandpermissions. Correspondence and requests for materials should be addressed to J.M.M. 